\title{
Novos sinônimos e ocorrências em Paspalum L. (Poaceae)
}

\author{
Regina Célia de Oliveira ${ }^{1,3}$ e José Francisco Montenegro Valls ${ }^{2}$
}

Recebido: 21.06.2006; aceito: 27.06.2008

ABSTRACT - (New synonyms and occurrences for Paspalum L. (Poaceae)). We propose fifteen synonyms for Paspalum species belonging to the informal Plicatula group: P. paludosum Swallen and P. validum Swallen to P. atratum Swallen, $P$. guaricense Swallen to $P$. centrale Chase, $P$. plicatulum Michx. var. villosissimum Pilg. to $P$. geminiflorum Steud., $P$. goyanum Swallen and $P$. plicatulum var. rigidum Döll to $P$. glaucescens Hack., $P$. gracile J. Le Conte and $P$. nicorae Parodi to $P$. lepton Schult., P. limbatum Henrard, P. formosum Swallen and P. pontanale Swallen to P. lenticulare Kunth, P. olivaceum Hitchc. \& Chase to $P$. riparium Nees, $P$. plicatulum var. cinereum Döll, $P$. macedoi Swallen and $P$. kempffii Killeen to $P$. rojasii Hack., and P. telmatum Swallen to P. wrightii Hitchc. \& Chase. Paspalum lepton is a new record for the Brazilian state of Mato Grosso do Sul.

Key words: Paniceae, Plicatula group, taxonomy

RESUMO - (Novos sinônimos e ocorrências em Paspalum L. (Poaceae)). Propõe-se a sinonimização de quinze nomes referentes a espécies de Paspalum, grupo informal Plicatula: P. paludosum Swallen e P. validum Swallen em P. atratum Swallen, $P$. guaricense Swallen em $P$. centrale Chase, $P$. plicatulum Michx. var. villosissimum Pilg. em $P$. geminiflorum Steud., $P$. goyanum Swallen e $P$. plicatulum var. rigidum Döll em $P$. glaucescens Hack., $P$. gracile J. Le Conte e $P$. nicorae Parodi em P. lepton Schult., $P$. limbatum Henrard, P. formosum Swallen e P. pontanale Swallen em P. lenticulare Kunth, $P$. olivaceum Hitchc. \& Chase em P. riparium Nees, P. plicatulum var. cinereum Döll, P. macedoi $\mathrm{S}$ wallen e P. kempffii Killeen em $P$. rojasii Hack. e $P$. telmatum Swallen em $P$. wrightii Hitchc. \& Chase. Relata-se pela primeira vez a ocorrência de $P$. lepton no estado brasileiro do Mato Grosso do Sul.

Palavras-chave: grupo Plicatula, Paniceae, taxonomia

\section{Introdução}

Paspalum engloba aproximadamente 400 espécies tropicais e subtropicais com ampla distribuição e diversidade no continente americano (Chase 1929). Estima-se que no Brasil ocorram 220 espécies com grande potencial forrageiro e que predominam em quase todas as formações campestres do país (Valls 1994).

Não há, até o presente, uma hipótese filogenética para o gênero em seu conjunto. No entanto, estudos preliminares sugerem que, para não ser mantido em parafilia, Paspalum deveria incluir as espécies de Thrasya Kunth (Giussani et al. 2001, Denham et al. 2002).

O gênero Paspalum caracteriza-se pelas inflorescências parciais racemiformes, espiguetas plano-convexas dispostas unilateralmente sobre a ráquis, com o dorso do lema superior em posição adaxial e, salvo algumas exceções, pela ausência da gluma inferior.

A subdivisão de Paspalum em dois subgêneros e mais de vinte grupos informais publicada por Chase (1929) e, posteriormente, ampliada (Chase, dados não publicados) tem sido amplamente utilizada pelos taxonomistas, às vezes com algumas modificações.

O presente trabalho é parte dos resultados obtidos na revisão taxonômica das espécies brasileiras do grupo Plicatula de Paspalum, ou seja, aquelas morfologicamente associadas a $P$. plicatulum Michx.

As cerca de 40 espécies de Paspalum grupo Plicatula são amplamente distribuídas no continente americano, à exceção de P. scrobiculatum L. e espécies relacionadas, que ocorrem também na Ásia, África e Oceania. Dentre os caracteres morfológicos das

1. Universidade Federal Rural do Semi-Árido (UFERSA), Herbário MOSS, Setor de Fitosanidade, BR-110, km 45, Caixa Postal 137, 59625-900 Mossoró, RN, Brasil

2. Embrapa Recursos Genéticos e Biotecnologia, Parque Estação Biológica - PqEB, Av. W5 Norte (final), Caixa Postal 02372, 70770900 Brasília, DF, Brasil

3. Autor para correspondência: reginacelia@ufersa.edu.br 
espécies de "Plicatula" utilizados por Chase (1929) e Barreto (1956) para descrever o grupo, destacamse os ramos unilaterais rígidos das inflorescências, as espiguetas com convexidade geralmente muito pronunciada, o lema inferior quase sempre com ondulações transversais e o antécio superior castanhoescuro e brilhante. Há relativo consenso entre os autores sobre as espécies que compõem o grupo.

O presente estudo fornece dados taxonômicos, nomenclaturais, informações sobre a distribuição geográfica, épocas de florescimento e frutificação e dados sobre ambiente, para cada espécie analisada.

\section{Material e métodos}

Foram revisados os herbários BAA, BHCB, BLA, CEN, CESJ, CTES, CPAP, ESAL, HB, IAN, IBGE, ICN, MBM, MG, MVFA, PACA, R, RB, SI, SP, SPF, UB, UEC e UPCB e, parcialmente, o herbário P. Os herbários HUEFS, INPA, IPA, UFG e UFMT enviaram materiais por empréstimo, sendo que, dos herbários G, MVFA, PEL, PH, B e US foram emprestados exemplares tipo, especificamente mencionados nas considerações abaixo sobre cada táxon, ou fornecido negativos, fotografias, fragmentos, imagems digitais e fotocópias de tipos, posteriormente incorporados ao acervo de herbário CEN. Os herbários são referidos pelos acrônimos, de acordo com Holmgren \& Holmgren (1998).

Os tipos cujo herbário de depósito não foi designado na obra original foram rastreados, a partir dos trabalhos de Chase \& Niles (1962), Stafleu \& Cowan (1976/1988) e no site http://www.mobot. mobot.org. Fragmentos de tipos, provenientes de diversos herbários europeus, catalogados e incorporados aos herbários SI, BAA e US, também auxiliaram na localização das exsicatas completas correspondentes.

Os dados de distribuição geográfica foram baseados em coletas, análises de herbários e na literatura.

\section{Resultados e Discussão}

Paspalum atratum Swallen, Phytologia 14(6): 378. 1967. TIPO: BRASIL. Mato Grosso do Sul: Campo Grande, 7-11-II-1930, A. Chase 10842 (holótipo US!).

= Paspalum paludosum Swallen, Phytologia 14(6): 379. 1967. TIPO: BRASIL. MARANHÃo: Carolina to San Antonio de Balsas, 20-25-III-1934, J.R. Swallen 3959 (holótipo US!), syn. nov.

= Paspalum validum Swallen, Phytologia 14(6): 382. 1967. TIPO: BRASIL. MARANHÃO: between Grajahú and Porto Franco, 8-13-III-1934, J.R. Swallen 3784 (holótipo US!; isótipo SP!), syn. nov.

Ocorre nas Américas Central e do Sul. No Brasil, foi encontrada nos estados do Amapá, Goiás, Maranhão, Mato Grosso, Mato Grosso do Sul, Minas Gerais, Pará, Roraima, São Paulo, Tocantins e no Distrito Federal. Habitam locais com solos argilosos, arenosos ou rochosos, úmidos ou secos. Encontrada com flores e frutos durante todo o ano.

O nome Paspalum paludosum foi relacionado a formas da espécie com pequeno porte, enquanto as linhagens típicas de $P$. atratum e $P$. validum atingem $2 \mathrm{~m}$ de altura. Porém, verificou-se que a altura das plantas analisadas é um caráter de variação contínua. Não foram encontrados caracteres que sustentassem a distinção dos táxons, nem mesmo a ornamentação do antécio superior observado em microscópio eletrônico de varredura (Oliveira 2004). Além disso, o holotipo de $P$. validum é extremamente semelhante ao de $P$. atratum.

Paspalum atratum é polimórfica e morfologicamente semelhante a $P$. plicatulum e $P$. lenticulare Kunth. Distingüe-se pelo colmo sem afilhamento nos nós superiores, bainhas foliares mais longas que os entrenós e largas em todo o comprimento, isto é, sem estreitamento na porção distal, de forma que os nós não ficam aparentes, lâminas (0,5-)1,2-2,7mm de largura e inflorescências com (3-)10-16 ramos unilaterais espiciformes. Paspalum limbatum pode atingir a mesma altura e possui espiguetas semelhantes às de P. atratum. Difere pelo intenso afilhamento nos nós superiores do colmo. Em P. plicatulum o colmo produz afilhos apenas na base, as bainhas podem ser ou não mais longas que os entrenós, mas possuem um estreitamento na porção distal, deixando os nós aparentes, as lâminas foliares mostram larguras que variam de $0,1-0,9 \mathrm{~mm}$ e as inflorescências apresentam 1-5 ramos.

Paspalum centrale Chase, J. Wash. Acad. Sci. 17: 145, f. 2. 1927. TIPO: EL SALVADOR. LA UnIón, 13-XI-1911, A.S. Hitchcock 8789 (holótipo US!). 
= Paspalum guaricense Swallen, Phytologia 14(6): 378. 1967. TIPO: VENEZUELA. El Dividive: Guárico, 11-IX-1927, H. Pittier 12520 (isótipo MO imagem digital!), syn. nov.

Ocorre no norte do Brasil e na América Central, avançando até as porções austrais da América do Norte. No Brasil, foi encontrada nos estados do Pará e Rondônia. Habita campos alagados e margens de estradas. Coletada com flores e frutos de fevereiro a agosto.

A análise de uma foto digital do isótipo de Paspalum guaricense e de outras exsicatas desta espécie provenientes da Venezuela permitiu-nos verificar que o nome foi aplicado a uma espécie já descrita anteriormente.

Paspalum centrale é relacionada a $P$. boscianum Flüggé. Diferem pela ráquis mais estreita, espiguetas de coloração olivácea, elípticas ou elíptico-ovais em $P$. centrale e ocráceas, amplamente obovais, raramente ovais ou elíptico-obovais em $P$. boscianum. Chase (1927) descreveu P. centrale como perene, porém, o holótipo é de uma planta anual. As gramíneas perenes são reconhecidas pelo número relativamente pequeno de eixos contendo flores, enquanto os demais continuam seu crescimento vegetativo nos anos seguintes. Além disso, geralmente possuem órgãos para a acumulação de reservas e propagação como, por exemplo, rizomas. As anuais, por sua vez, têm grande número de colmos floríferos e ausência de órgãos para acumulação de reservas e propagação (Rua \& Gróttola 1997).

Paspalum geminiflorum Steud., Syn. Pl. Glumac. 1: 25. 1855 (1853). TIPO: BRASIL. Minas GERAIS: s. local, s.d., Clausen 1021 (holótipo P n.v., frag. US!).

= Paspalum plicatulum var. villosissimum Pilg. ("villosissima" na publicação original), Bot. Jahrb. Syst. 30(1): 131. 1901. TIPO: BRASIL. Mato Grosso: "auf trockenen Wiesen im Cuyabáthal oberhalb von Rosario", IV-1899, R. Pilger 440 (Holótipo B n.v., frag. US!; isótipo B n.v., frag. BAA!), syn. nov.

Ocorre do norte da América do Sul até o sudeste do Brasil. No Brasil, foi encontrada nos estados do Amazonas, Goiás, Maranhão, Mato Grosso, Minas Gerais, Pará, São Paulo e no Distrito Federal. Habita áreas campestres com presença de componentes arbóreos em solos pedregosos ou lateríticos. Encontrada com flores e frutos praticamente durante todo o ano.
Caracteriza-se pelo hábito perene e ornamentação reticular da gluma superior e lema inferior. Esta ornamentação característica ocorre também no holótipo de Paspalum plicatulum var. villosissimum, o qual também é semelhante a $P$. geminiflorum nas demais características, permitindo-nos a proposição deste sinônimo.

Paspalum glaucescens Hack., Oesterr. Bot. Z. 51: 237. 1901. TIPO: BRASIL. MINAS GERAIS: "prés Ouro Preto", 9-V-1892, Glaziou 20130 (holótipo W n.v., frag. US!; isótipo P!, frag US! e foto $\mathrm{SI} !)$.

= Paspalum goyanum Swallen, Phytologia 14(6): 376. 1967. TIPO: BRASIL. GoIÁs: between Jatahy and Rio Araguaya, $17^{\circ} 40^{\prime}$ to $16^{\circ} 50^{\prime} \mathrm{S}, 51^{\circ} 40^{\prime}$ to $53^{\circ} \mathrm{W}, 3-\mathrm{IV}-1930$, A. Chase 11733 (holótipo US!), syn. nov.

= Paspalum plicatulum var. rigidum Döll in Mart., Fl. Bras. 2(2): 77. 1877. TIPO: BRASIL. MinAS GERAIS: Curvello, III-1835, Lund s.n. (holótipo P n.v., frag. US!), syn. nov.

Centro e sul do Brasil, Paraguai, nordeste da Argentina e Uruguai (Barreto 1956, Zuloaga \& Morrone 2005). No Brasil, ocorre nos estados de Goiás, Mato Grosso, Mato Grosso do Sul, Minas Gerais, Paraná, Rio Grande do Sul, Santa Catarina, São Paulo e no Distrito Federal. Habita preferencialmente savanas com solos mal drenados.

Um dos caracteres diagnósticos de Paspalum glaucescens é a lâmina foliar filiforme na porção proximal e plana na distal, o que confere um aspecto de lâmina peciolada. O holótipo de $P$. goyanum é uma planta com lâminas foliares totalmente filiformes, e este era o principal caráter diferencial entre as duas. No entanto, encontramos variação em um mesmo indivíduo (como pode ser analisado entre algumas duplicatas de Valls et al. 13942 (CEN), ou seja, indivíduos com lâminas totalmente filiformes e outras com a porção distal plana e a proximal filiforme. A variação na forma da lâmina foliar pode estar relacionada à rebrota, ou seja, aos estágios iniciais de desenvolvimento das lâminas após a remoção por corte, pastejo ou queimadas.

O fragmento do tipo de Paspalum plicatulum var. rigidum consta apenas de espiguetas as quais, são idênticas às de $P$. glaucescens. As espiguetas de $P$. glauscescens representam outro caráter diagnóstico da 
espécie, são elíptico-obovais, com convexidade pouco pronunciada, e três nervuras conspícuas, distribuídas de forma eqüidistante, no dorso da gluma superior.

Ao contrário do que tem sido observado na maioria das espécies de Paspalum, onde predominam linhagens tetraplóides apomíticas (Quarín 1992), P. glaucescens apresenta uma alta proporção de plantas diplóides (Pozzobon \& Valls 2000, Pozzobon et al. 2000). Nas espécies com linhagens tetraplóides apomíticas, as plantas diplóides têm-se mostrado sempre sexuais alógamas (Quarín 1992), o que faz prever grande heterogeneidade em suas progênies, aspecto que, aparentemente, tem favorecido o surgimento de muitos sinônimos, pela descrição de meros segmentos dessa ampla variação morfológica como espécies distintas (Pozzobon \& Valls 2000).

Paspalum lenticulare Kunth, Nov. Gen. Sp. 1: 92. 1815. TIPO: VENEZUELA. SUCRE, IX, F.W.H.A. von Humboldt \& A.J.A. Bonpland s.n. (isótipo LE n.v., frag.!).

= Paspalum limbatum Henrard, Blumea 4(3): 511. 1941. TIPO: PARAGUAI. GuAIRÁ: Villa Rica, 10X-1874, B. Balansa 107 (isótipo US!), syn. nov.

= Paspalum formosum Swallen, Phytologia 14(6): 378. 1967. TIPO: BRASIL. Minas Gerais: Ituiutaba, 18-II-1951, A. Macedo 3171 (holótipo US!; isótipo SP!), syn. nov.

= Paspalum pontanale Swallen (“pontanalis" na publicação original), Phytologia 14(6): 376. 1967. TIPO: BRASIL. Mato Grosso (do Sul): Porto Esperança, 28-II-1930, A. Chase 11080 (holótipo US!), syn. nov.

Ocorre na América Central e do Sul. No Brasil, está documentada nos estados do Acre, Alagoas, Amapá, Goiás, Mato Grosso, Mato Grosso do Sul, Minas Gerais, Pará, Rio de Janeiro, Rio Grande do Sul, São Paulo, Rondônia, Tocantins e no Distrito Federal. Habita solos argilosos e, mais raramente, areno-argilosos ou arenosos, em campo úmido, eventualmente próximo a carandazais ou a veredas. Domina em algumas formações do Pantanal Mato-grossense. Encontrada com flores e frutos durante todo o ano.

Quarín (1975), Killeen (1990), Renvoize (1988) e Oliveira \& Valls (2001) trataram desta espécie com distintas delimitações e aceitaram algumas espécies agora propostas como sinônimos. Ao avaliar a variação morfológica de Paspalum lenticulare, ao longo da ampla área de ocorrência da espécie, chegouse à conclusão que os nomes aqui propostos como sinônimos se aplicam a extremos morfológicos de uma variação contínua, principalmente relativos à largura da folha e ao comprimento e largura das espiguetas. A largura das lâminas foliares de $P$. lenticulare, como aqui delimitada, varia de $0,1-1,5 \mathrm{~cm}$ e as espiguetas de 2-2,8(-3,1) x 1,1-1,9mm.

Paspalum lenticulare é reconhecida vegetativamente pelos colmos multinodes muito ramificados na porção distal.

Paspalum lepton Schult., Mant. 2: $173.1824 \equiv P$. gracile J. Le Conte, J. Phys. Chim. Hist. Nat. Arts 91: 285. 1820, nom. illeg., non Rudge (1805), nec Poiret (1816), nec Schletendal (1854). TIPO: ESTADOS UNIDOS. GEÓRgIA: s. local, s.d., $s$. col. (provavelmente H.C. Muhlenberg) (holótipo $\mathrm{PH}$ fotocópia!).

= Paspalum nicorae Parodi, Notas Mus. La Plata, Bot. 8 (40): 82. 1943 三 Paspalum plicatulum var. arenarium Arechav., Anales Mus. Nac. Montevideo 1: 58. 1894. TIPO: URUGUAI. Río de la Plata, III-IV, Arechavaleta s.n. (isótipo US!), syn. nov.

Ocorre no Uruguai, Paraguai, Argentina, Brasil e Estados Unidos, onde pode ter sido difundida com o gado proveniente da América do Sul (Barreto 1956). No Brasil, foi encontrada nos estados do Rio Grande do Sul, Santa Catarina e Mato Grosso do Sul. O material de São Paulo é proveniente de cultivo e os materiais citados por Oliveira \& Valls (2001), com dúvidas, sob $P$. nicorae, foram posteriormente incluídos na circunscrição de $P$. plicatulum (Oliveira 2004). Esta é a primeira citação de P. lepton para o Mato Grosso do Sul.

O nome Paspalum nicorae tem sido amplamente utilizado (Barreto 1956, Burkart 1969, Cabrera 1970, Rosengurtt et al. 1970, Smith et al. 1982, Oliveira \& Valls 2001), mas os rizomas leptomorfos característicos da espécie são muito evidentes na fotocópia do holótipo de P. lepton, o que levou à presente proposta de sinonimização.

Habita locais com solos arenosos secos ou mal drenados.

Relaciona-se morfologicamente a Paspalum rhodopedum L.B. Sm. \& Wassh. e a P. plicatulum. Distingue-se de $P$. plicatulum pelos rizomas 
leptomorfos. Paspalum rhodopedum diferencia-se por apresentar indivíduos robustos, com rizomas de entrenós curtos e grossos, lâminas freqüientemente com um estreitamento proximal e coloração avermelhada na superfície abaxial, com nervura central saliente além de espiguetas geralmente obovais. Plantas típicas de $P$. lepton são delicadas e formam touceiras de folhagem glauca, com rizomas leptomorfos longos, comprimidos na porção proximal, lâminas foliares com nervura central de coloração mais clara e espiguetas elíptico-ovais.

Paspalum riparium Nees in Mart., Fl. Bras. Enum. Pl. 2: 56. 1829. TIPO: BRASIL. Amazonas: "Fluminis Nigri passim", XI-1819, K.F.P. von Martius s.n. (holótipo M n.v., frag. US!).

= Paspalum olivaceum Hitchc. \& Chase, Contr. U. S. Natl. Herb. 18(7): 310. 1917. TIPO: GuADELOUPE: Habitation convenance (Bahie Mahaut), 23-IX1897, A. Duss 3915 (isótipo NY imagem digital!), syn. nov.

Ocorre no Brasil, Guianas, Venezuela, Colômbia e América Central. No Brasil, foi encontrada nos estados do Acre, Amazonas, Amapá, Ceará, Maranhão, Mato Grosso, Pará, Piauí, Rondônia e Roraima. Habita solos arenosos nas margens de rios e locais antropizados. Encontrada com flores e frutos em todos os meses do ano.

Paspalum riparium é uma espécie anual, muito característica pelas lâminas foliares pruinosas, espiguetas elípticas e lema inferior com as margens alvas e a porção central evidenciando a coloração atropurpúrea do antécio superior. A imagem digital do isótipo de P. olivaceum mostra todos esses caracteres, o que justifica a sinonímia proposta.

Paspalum rojasii Hack., Repert. Sp. Nov. Regni Veg. 7: 369. 1909. TIPO: PARAGUAI. "in campis siccis prope Estrella”, I-1908, T. Rojas 10122 (isótipo BM!, isótipo MVFA n.v., US frag.!) $\equiv$ Paspalum guenoarum Arechav. var. rojasii (Hack.) Parodi ex Burkart, Fl. Il. Entre Ríos 6(2): 389. 1969.

= Paspalum plicatulum var. cinereum Döll in Mart., Fl. Bras. 2(2): 77. 1877. TIPO: BRASIL. s. local, s. data, L.C.M. Richard s.n. (holótipo B n.v., frag. US!), syn. nov.

= Paspalum macedoi Swallen, Phytologia 14(6): 377 . 1967. TIPO: BRASIL. Minas Gerais: Monte
Alegre, 10-II-1956, A. Macedo 4299 (holótipo US!; isótipo CPATU!), syn. nov.

= Paspalum kempffii Killeen, Ann. Missouri Bot. Gard. 77(1): 179, f5. 1990. TIPO: BOLÍVIA. SANTA CRuZ: Ñuflo de Chávez, $16^{\circ} 08^{\prime} \mathrm{S}, 62^{\circ} 05^{\prime} \mathrm{W}$, Estancia La Pachanga, $5 \mathrm{~km} \mathrm{~S}$ of Concepción, 3-I-1987, T.J. Killeen 2272 (isótipos MO imagem digital!, US!, SI!, CTES!), syn. nov.

Espécie descrita do Paraguai, também ocorre no Brasil e na Bolívia, tendo sido citada para este último país por Killeen (1990) e Renvoize (1998), sob P. kempffii e P. macedoi, respectivamente. No Brasil, ocorre nos estados de Alagoas, Bahia, Goiás, Minas Gerais, Paraná, Paraíba, Rio Grande do Sul, Santa Catarina, São Paulo e no Distrito Federal. Habita savanas arenosas, sendo ocasional em campos rupestres. Encontrada com flores e frutos durante quase todo o ano, exceto nos meses de agosto e setembro. O material cultivado pelos autores em telado na Embrapa Recursos Genéticos e Biotecnologia, em Brasília, Distrito Federal, floresceu e frutificou em agosto e setembro.

Embora Paspalum rojasii apresente pouca afinidade morfológica com P. guenoarum Arechav., estas espécies têm sido associadas por vários autores. Segundo Quarín (dados não publicados), o "Pasto Ramirez" e o "Pasto Rojas" são linhagens de $P$. guenoarum morfologicamente muito parecidas. De acordo com Quarín, o fato de o "Pasto Rojas" ter recebido este nome vulgar levou à interpretação errônea de que corresponderia a $P$. rojasii, surgindo daí a confusão entre os agrostólogos regionais. Os materiais A.G. Schulz 12207 e 15822, depositados no herbário CTES, foram colhidos na Estação Experimental de Colonia Benítez sob "Pasto Rojas", e o exemplar A.G. Schulz 15821, do mesmo local, sob "Pasto Ramirez". Todavia, os três exemplares se enquadram em $P$. guenoarum. Talvez esta confusão tenha estimulado o surgimento dos novos nomes, que agora estão sendo sinonimizados com $P$. rojasii. Esta espécie mostra grande variação no tamanho (2,8-4 x 2,1-7 mm) e na forma (obovais ou elíptico-obovais) das espiguetas.

Paspalum rojasii possui colmos com 3-4 nós, lâmina foliar conduplicada na porção proximal e plana na distal, o que confere um aspecto de lâmina peciolada ou, raramente, totalmente conduplicadas. Paspalum guenoarum possui colmos de 5-6(-8) nós e lâminas foliares planas, sem pseudo-peciolo. A gluma 
superior de Paspalum rojasii é da mesma largura do antécio superior e em $P$. guenoarum é mais larga, ficando "frouxa" na espigueta.

Zuloaga \& Morrone (2003) propuseram a sinonimização de Paspalum kempffii e P. macedoi a $P$. guenoarum e a de $P$. rhodopedum em $P$. guenoarum var. rojasii, mantendo esta posição em Zuloaga \& Morrone (2005). De fato, P. rojasii é muito semelhante a $P$. rhodopedum, mas esta última espécie apresenta rizomas leptomorfos longos e o rizoma de $P$. rojasii é curto, inconspícuo. Materiais vivos de ambas espécies estão sendo mantidas em telado, na Embrapa Recursos Genéticos e Biotecnologia. Com isto, foi possível observar as porções subterrâneas de ambas em vários períodos do ano e verificar que não há formação de rizomas longos, em $P$. rojasii.

Paspalum wrightii Hitchc. \& Chase, Contr. U.S. Natl. Herb. 18(7): 310. 1917. TIPO: CUBA. PINAR DEL Río: Pinar del Río, s.d., C. Wright 3843 (holótipo US n.v., frag. MVFA!).

$=$ Paspalum telmatum Swallen, Phytologia 14(6): 388 . 1967. TIPO: BRASIL. Mato Grosso do Sul: Campo Grande - Dourados, Lagoinha, 14-17-II1930, A. Chase 10926 (holótipo US!), syn. nov.

Com a inclusão de Paspalum hydrophilum Henrard na circunscrição de $P$. wrightii (Zuloaga \& Morrone 2003), foi ampliada a distribuição conhecida da espécie - que se estende do sul do Brasil, noroeste da Argentina e Paraguai (Martínez \& Quarín 1999) até os Estados Unidos. No Brasil, $P$. wrightii ocorre nos Estados de Goiás, Mato Grosso, Mato Grosso do Sul, Rondônia e Pará. Pozzobon \& Valls (2003) relataram a grande freqüência de $P$. wrightii (sob $P$. hydrophilum) nas áreas periodicamente inundadas do Pantanal Mato-grossense. Encontrada com flores e frutos de novembro a abril.

As diferenças apresentadas pelo holótipo de Paspalum telmatum em relação ao de $P$. wrightii - lâminas velutíneas em $P$. telmatum e geralmente glabras ou levemente pilosas em $P$. wrightii, gluma superior e lema inferior muito mais longo que o antécio superior em $P$. telmatum que em $P$. wrightii e antécio e espigueta com coloração mais clara em P. telmatum - ocorrem, em diferentes graus, entre os distintos indivíduos examinados da espécie. Como P. wrightii apresenta três níveis de ploidia, com a possibilidade de apomixia ou sexualidade (Norrmann 1981, Pozzobon \& Valls 2003) pode-se esperar grande variabilidade nas progênies desta espécie.

Zuloaga \& Morrone (2005) consideram Paspalum telmatum como pertencente ao grupo Livida. Embora a coloração pouco intensa do antécio superior do exemplar tipo possibilite tal interpretação, as demais características morfológicas enquadram-se muito bem na variação mostrada por $P$. wrightii.

Paspalum wrightiié relacionada morfologicamente a Paspalum modestum Mez e P. palustre Mez (Martínez \& Quarín 1999, Pozzobon \& Valls 2003). Compartilham o hábito perene, a preferência por ambientes úmidos, apresentam bainhas aerenquimatosas, nervura mediana da lâmina foliar de coloração mais clara, ráquis alada e espiguetas ferrugíneas. Paspalum wrightii é distinguida pelos longos rizomas com catáfilos desenvolvidos.

\section{Agradecimentos}

À CAPES, pela concessão da bolsa de doutorado e ao CNPq, pelo apoio financeiro e bolsa de Produtividade em Pesquisa ao segundo autor. Agradecemos também aos curadores dos herbários, por facilitarem e disponibilizarem os materiais para nossos estudos.

\section{Literatura citada}

Barreto, I.L. 1956. Las especies afines a Paspalum plicatulum en Rio Grande del Sur (Brasil). Revista Argentina de Agronomía 23: 53-70.

Burkart, A. 1969. Paspalum. In: A. Burkart (ed.). Flora Ilustrada de Entre Ríos (Argentina). Parte II (Gramíneas). INTA, Buenos Aires, v.6, parte 2, pp. 369-411.

Cabrera, A.L. 1970. Paspalum. In: A.L. Cabrera (ed.). Flora de la Provincia de Buenos Aires. Parte II (Gramíneas). INTA, Buenos Aires, v. 4, parte 2, pp. 518-537.

Chase, A. 1927. New Grasses. Journal of the Washington Academy of Sciences 17: 145.

Chase, A. 1929. The North American species of Paspalum. Contributions from the United States National Herbarium 28: 1-310.

Chase, A. \& Niles, C.D. 1962. Index to grass species, v.3. G.K. Hall, Massachusetts.

Denham, S.S., Zuloaga, F.O. \& Morrone, O. 2002. Systematic revision and phylogeny of Paspalum subgenus Ceresia (Poaceae: Panicoideae: Paniceae). Annals of the Missouri Botanical Garden 89: 337399. 
Giussani, L.M., Cota-Sánchez, J.H., Zuloaga, F.O. \& Kellogg, E.A. 2001. A molecular phylogeny of the Grass Subfamily Panicoideae (Poaceae) shows multiple origins of $\mathrm{C}_{4}$ photosynthesis. American Journal of Botany 88: 1993-2012.

Holmgren, P. K. \& Holmgren, H. 1998 [continuously updated]. Index Herbariorum: A global directory of public herbaria and associated staff. New York Botanical Garden's Virtual Herbarium. http://sweetgum.nybg.org/ ih/ (acesso em 23.03.2005).

Killeen, T.J. 1990. The grasses of Chiquitanía, Santa Cruz, Bolivia. Annals of the Missouri Botanical Garden 77: 125-201.

Martínez, E.J. \& Quarín, C.L. 1999. Citoembriología y comportamiento reproductivo de un citotipo diploide de Paspalum hydrophilum y sus híbridos com P. palustre (Poaceae, Paniceae). Darwiniana 37: 243-251.

Norrmann, G. 1981. Citología y método de reproducción en dos especies de Paspalum (Gramineae). Bonplandia 5: $149-158$

Oliveira, R.C. 2004. O gênero Paspalum L., grupo Plicatula (Poaceae: Paniceae), no Brasil. Tese de Doutorado, Universidade Estadual de Campinas, Campinas.

Oliveira, R.C. \& Valls, J.F.M. 2001. Paspalum. In: M.G.L. Wanderley, G.J. Shepherd \& A.M. Giulietti (coords.). Flora Fanerogâmica do Estado de São Paulo. V.1 (Poaceae). Hucitec, São Paulo, pp. 191-228.

Pozzobon, M.T. \& Valls, J.F.M. 2000. Cytogeography and variation of stomatal size of Paspalum glaucescens (Gramineae; Paniceae) in Southern Brazil. Euphytica 116: 251-256.

Pozzobon, M.T. \& Valls, J.F.M. 2003. Chromosome number in Brazilian germoplasm accessions of Paspalum hydrophilum, $P$. modestum and $P$. palustre (Gramineae; Paniceae). Genetics and Molecular Biology 26: 365-368.

Pozzobon, M.T., Valls, J.F.M. \& Santos, S. 2000. Contagens cromossômicas em espécies brasileiras de
Paspalum L. (Gramineae). Acta Botanica Brasilica 14: 151-162.

Quarín, C.L. 1975. Notas sobre el género Paspalum (Gramineae). Bonplandia 3: 195-210.

Quarín, C.L. 1992. The nature of apomixis and its origin in Panicoid grasses. Apomixis Newsletter, special issue $1^{\text {st }}$ Aponet Workshop Montpellier 5: 8-15.

Renvoize, S.A. 1988. Gramíneas de Bolivia. Royal Botanic Gardens, Kew.

Rosengurtt, B., Arrillaga-de-Maffei, B. \& Izaguirre-deArtucio, P. 1970. Gramíneas Uruguayas. Universidad de la Republica, Montevideo.

Rua, G.H. \& Gróttola, C.M. 1997. Growth form models within the genus Paspalum L. (Poaceae, Paniceae). Flora 192: 65-80.

Smith, L.B., Wasshausen, D.C. \& Klein, R.M. 1982. Gramíneas. Gêneros: Paspalum até Zea. In: R. Reitz (ed.). Flora Ilustrada Catarinense. Herbário Barbosa Rodrigues, Itajaí, v. 3, pp. 911-1407.

Stafleu, F.A. \& Cowan, R.S. 1976/1988. Taxonomic Literature 2 ed., 7 v., Bohn, Scheltema \& Holkema, Utrech.

Valls, J.F.M. 1994. O potencial de plantas forrageiras tropicais americanas. Anais do Simpósio Brasileiro de Forrageiras e Pastagens. CBNA, Campinas, pp.11-24.

Zuloaga, F.O. \& Morrone, O. 2003. Paspalum. In: R.J. Soreng \& S.J. Pennington (eds.). Catalogue of New World Grasses (Poaceae): III. Subfamilies Panicoideae, Aristidoideae, Arundinoideae, and Danthonioideae. Contributions from the United States National Herbarium 46: 443-527.

Zuloaga, F.O. \& Morrone, O. 2005. Revisión de las especies de Paspalum para América del Sur austral (Argentina, Bolívia, Sur del Brasil, Chile, Paraguay y Uruguay). Missouri Botanical Garden Press, St. Louis. 